\title{
Evaluation of Anti-urolithiatic Potential of Leaves of Alstonia scholaris and its Isolated Pentacyclic Triterpenoids in Ethylene Glycol-induced Renal Calculi Rat Model
}

\author{
Zehra S and Sanaye MM* \\ Department of Pharmacology and Toxicology, Prin. K. M. Kundnani College of Pharmacy, Mumbai, Maharashtra, INDIA.
}

\begin{abstract}
Introduction: Urolithiasis has afflicted human race since the days of yore. Conventional treatment acts only on associated ailments whereas pharmacologically active phytoconstituents derived from plants are reported to possess lithotriptic as well as anti-urolithiatic effect, thus herbal sources need to be scientifically explored. Present study was aimed to evaluate anti-urolithiatic potential of leaves of Alstonia scholaris and its isolated pentacyclic triterpenoids: lupeol and ursolic acid in ethylene glycolinduced renal calculi rat model. Materials and Methods: Leaves of Alstonia scholaris were extracted with various solvents, amount of triterpenoids in each extract was quantified using HPTLC. Extract which had higher amount of triterpenoid was evaluated for its anti-urolithiatic effect. Albino wistar rats of either sex were divided into 6 groups. All groups except vehicle were fed with $0.75 \% \mathrm{v} / \mathrm{v}$ of ethylene glycol for 28 days. Treatment with standard cystone, low and high dose of extract and pentacyclic triterpenoids were given to respective groups from $4^{\text {th }}-28^{\text {th }}$ day of study. On $28^{\text {th }}$ day, serum creatinine, uric acid, BUN, TNF- $\alpha$, IL- 6 and urinary electrolyte levels were estimated. Kidneys were isolated, one was used for histopathological study and other for antioxidant assays. Results and Discussion: Histopathological examination of kidneys revealed destructed morphology due to deposition of large polymorphic crystals and cystic dilatation of tubules. Whereas treatment with extract and its pentacyclic triterpenoids prevented renal damage and deposition of crystals. Perturbations in levels of nitrogenous wastes, inflammatory mediators and urinary electrolytes were also prevented. Alstonia scholaris rich in triterpenoid significantly arrested associated oxidative stress and inflammation thus preventing formation of renal calculi. Conclusion: Leaves of Alstonia scholaris and pentacyclic triterpenoids isolated from them demonstrates potent herbal remedy for treatment of urolithiasis.
\end{abstract}

Key words: Alstonia scholaris, Anti-inflammatory, Lupeol, Oxidative stress, Ursolic acid, Urolithiasis.

\section{INTRODUCTION}

Urolithiasis has afflicted mankind since long time. It is characterized by calcifications in urinary system, usually in kidneys or ureters, but may also affect bladder or urethra. Approximately, $12 \%$ of the world's population is estimated to be suffering from it. ${ }^{1}$ Although, there are different types of renal calculi named after their chemical composition like calcium oxalate $(\mathrm{CaOx})$, calcium phosphate, uric acid, struvite, cysteine, etc; $\mathrm{CaOx}$ is the most common component and represents $80 \%$ of analyzed stones. $^{2}$

Open renal surgery has become obsolete and is rarely performed now-a-days, since introduction of Extracorporeal Shockwave Lithotripsy (ESWL) which breaks down staghorn calculi into small to medium
Submission Date: 01-11-2019; Revision Date: 12-04-2020; Accepted Date: 11-07-2020

DOI: 10.5530/ijper.55.1.26 Correspondence: Dr. Mrinal M. Sanaye Pharmacology and Toxicology, Associate Professor in Pharmacology, Prin. KM Kundnani College of Pharmacy Mumbai- 400005, Maharashtra, INDIA. Phone no: +91 09820270269 Email id: msmrinalms@ gmail.com

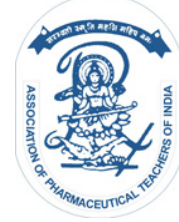

www.ijper.org 
sized stones which eventually passes out in urine spontaneously or with Medical Expulsive Therapy (MET). But traumatic effects of these shockwaves and residual stone fragments left behind results in acute renal injury, increased renal infection and stone recurrence. ${ }^{3}$ Also, MET carries risk of side effects such as severe orthostatic hypotension and allergic reactions thus limiting therapy for not more than 6 weeks. This indicates that current medical management of urolithiasis is costly, time bound and associated with substantial side effects. Another major problem associated with urolithiasis is recurrence. The recurrence rate after encountering the first episode is reported to be as high as $50 \%$ after 5 years and $80-90 \%$ after 10 years. ${ }^{4}$ Thus indicating prevention of the condition to be of prime importance. Thus, there is a need to explore preventive alternative therapy which will be safe, effective and economical.

Pentacyclic triterpenoids are claimed to be efficient in preventing $\mathrm{CaOx}$ calculus-induced renal per-oxidative damage and dilution of urinary stone forming constituents. ${ }^{5}$ Thus, plant rich in pentacyclic triterpenoids could carry promising therapeutic potential to target urolithiasis. Leaves of Alstonia scholaris (Family: Apocynaceae; Common name: Saptaparni) are rich with pentacyclic triterpenoids like lupeol, betulin and ursolic acid. ${ }^{6}$ In present study, leaf extract of $A$. scholaris and pentacyclic triterpenoids viz. lupeol and ursolic acid isolated from extract are assessed for anti-urolithiatic effect.

\section{MATERIALS AND METHODS}

\section{Plant}

The fresh leaves of $A$. scholaris were procured from Joginder Nursery, Delhi and authenticated at St Xavier's Blatter Herbarium (Mumbai) under specimen number NI-1417 of N. A. Irani. Leaves were shade dried and crushed to obtain a coarse powder, which was subjected to soxhlet extraction using solvents such as methanol (MeAS), chloroform (ChAS), ethanol (EtAS) and hydroethanol (HeAS) (70:30-Ethanol:Water). After extraction, excess of solvent from each extract was evaporated on electronic water bath to obtain semi-solid mass, which was stored in airtight container at $2-8^{\circ} \mathrm{C}$.

\section{Chemicals}

Ethylene glycol (EG) and ammonium chloride were procured from SD Fine-Chem Ltd (Mumbai, India). Cystone was purchased from Himalaya Drug Company (Bangalore, India.). Biochemical kits were procured from Erba Mannheim (Transasia, Germany) and Crest biosystems Ltd. ELISA kits were purchased from Krishgen biosystems. All the other chemicals used were of analytical grade and purchased from approved chemical suppliers.

\section{Estimation of triterpenoids by HPTLC method}

$100 \mathrm{mg}$ each of MeAS, ChAS, EtAS and HeAS were accurately weighed and transferred to $10 \mathrm{ml}$ volumetric flasks, making up the volume with methanol. This was sonicated for $900 \mathrm{~s}$ and used as stock solution.

Aluminium silica gel $60 \mathrm{~F}_{254}$ TLC plates (Merck, USA) were cut in $10 \times 10 \mathrm{~cm} .8 \mathrm{~mm}$ bands of $5 \mu \mathrm{L}$ aliquot was applied by Camag Linomat V applicator. Twin trough developing chamber was saturated with optimized mobile phase n-Hexane: Ethyl acetate $(5: 5 \mathrm{v} / \mathrm{v})$ for 20 $\mathrm{m}$ after which the plate was developed. The developed plate was air dried and derivatized using freshly prepared Anisaldehyde Sulphuric acid Reagent (ASR). After derivatization, plate was scanned at $540 \mathrm{~nm}$ in Camag TLC Scanner equipped with vision CATS software.

\section{Isolation of pentacyclic triterpenoids}

Pentacyclic triterpenoids viz. Lupeol and Ursolic acid were isolated from EtAS by Preparative-TLC method. The isolated compounds were characterized by IR spectroscopy and Mass spectrometry.

\section{Animals}

Experimental protocol (KMKCP/IAEC/10/2017) was approved by the Institutional Animal Ethics Committee (IAEC) and work was performed as per National Institute of Health (NIH) guidelines. Albino wistar rats of either sex weighing 150-180 gm were procured from registered animal breeder; Bharat Serum and Vaccines Limited, Thane (CPCSEA Reg. No: 103/PO/RcBiBt-S/NRcL/99/CPCSEA). Animals were maintained in hygienic condition in clean plastic cages containing corn cob bedding. They were fed with standard rat pellet diet and supplied with water ad libitum. Animal house (CPCSEA Reg. No: 25/PO/ReBi/S/99/CPCSEA) was maintained under standard environmental conditions of temperature $\left(24 \pm 1^{\circ} \mathrm{C}\right)$ and relative humidity $(65 \% \pm 10 \%) .12 \mathrm{~h}$ light and dark cycle was followed. The animals were allowed to acclimatize to animal house conditions for 6-7 days prior to the experiment.

\section{Experimental design}

Ethylene glycol (EG) induced urolithiasis method was used to assess anti-urolithiatic activity in rats. ${ }^{7} 36$ albino wistar rats of either sex weighing 150-180 gm were divided into 6 groups. Group I: Vehicle Control group (VC) was administered with CMC $(1 \% \mathrm{w} / \mathrm{v})$ and distilled water $(2 \mathrm{ml} / \mathrm{kg})$, p.o for 28 days. Group II: Calculi induced group (CI); Group III: Standard treatment 
group (TT/CYS); Group IV: Low dose treatment group (TT/LD); Group V: High dose treatment group (TT/HD); Group VI: Pentacyclic Triterpenoids treatment group (TT/PT) were administered with $0.75 \% \mathrm{v} / \mathrm{v}$ of EG for 28 days and $1 \% \mathrm{w} / \mathrm{w}$ of ammonium chloride for initial 3 days in drinking water. (Ammonium chloride helps in accelerating urolithiasis). Additionally, Groups III, IV, V, VI were treated with Standard Cystone $(400 \mathrm{mg} / \mathrm{kg}$ b.w, p.o), EtAS (200 mg/kg b.w, p.o), EtAS (400 mg/ $\mathrm{kg}$ b.w, p.o), lupeol $(1.24 \mathrm{mg} / \mathrm{kg}$ b.w, p.o) + ursolic acid $\left(9.36 \mathrm{mg} / \mathrm{kg}\right.$ b.w, p.o) respectively from $4^{\text {th }}$ to $28^{\text {th }}$ day of study.

\section{Assessment of anti-urolithiatic activity}

\section{Serum collection and analysis}

On the $28^{\text {th }}$ day, $1-2 \mathrm{ml}$ of blood samples were withdrawn from retro orbital plexus of rats under light anesthesia, blood samples were centrifuged (Remi R-4c DX) at 2000-2500 rpm for $15 \mathrm{~m}$ to obtain clear serum which were analyzed for presence of Glomerular Filtration Rate (GFR) markers such as creatinine, uric acid and BUN using ERBA diagnostic kits and biochemical analyzer-ERBA chem-7 (Transasia, Germany). Inflammatory mediator levels were estimated using rat TNF- $\alpha$ and IL-6 ELISA kits, microplate reader-ELx800 and microplate washer-ELx50 (Biotek Instruments).

\section{Urine collection and analysis}

$24 \mathrm{~h}$ urine samples were collected by placing each rat in individual metabolic cage on $28^{\text {th }}$ day with free access to drinking water during urine collection period. Urine samples were analyzed for presence of electrolytes such as sodium, potassium, chloride, calcium and phosphorus using diagnostic kits from Crest and ERBA. Oxalate content was determined by colorimetric method. ${ }^{8}$

\section{Microscopic examination of urine for crystals}

Collected urine samples were centrifuged at 2000-2500 $\mathrm{rpm}$ for $10 \mathrm{~m}$. $1 \mathrm{ml}$ of urine was discarded and the sediment was remixed in the remaining half volume to get concentrated urine solution. A drop of this solution was placed on the glass slide and observed under light microscope at $45 \mathrm{X}$.

\section{Isolation of kidneys}

Animals were sacrificed in carbon dioxide chamber. The abdomen was cut open to remove both kidneys. Kidneys were cleaned off from extraneous tissues, weight was recorded and preserved in 10\% Neutral Buffered Formalin (NBF) at $2-8^{\circ} \mathrm{C}$. One kidney was used for histopathological studies and other for estimation of tissue parameters.

\section{Histopathological examination}

The kidneys were processed, embedded in paraffin, sectioned and stained with H\&E stain and observed under light microscope (magnification: X400) for presence of calculi and morphological changes.

\section{Kidney tissue homogenate}

Kidneys were dried at $80^{\circ} \mathrm{C}$ in hot air oven. A sample of $100 \mathrm{mg}$ of dried kidney was boiled in $10 \mathrm{ml}$ of $1 \mathrm{~N}$ $\mathrm{HCl}$ for $30 \mathrm{~m}$ and homogenized. The homogenate was centrifuged at $2000-2500 \mathrm{rpm}$ for $10 \mathrm{~m}$ and the supernatant was separated. ${ }^{9}$ Calcium and oxalate content of kidney tissue were determined. Levels of antioxidant enzymes like superoxide dismutase $(\mathrm{SOD}){ }^{10}$ catalase $(\mathrm{CAT})^{11}$ reduced glutathione $(\mathrm{GSH})^{12}$ and malondialdehyde $(\mathrm{MDA})^{13}$ were estimated by UV Spectrophotometer. (Jasco UV-350). TNF- $\alpha$ and IL-6 levels were also determined.

\section{Statistical analysis}

Results were expressed as Mean \pm S.D. Data was analyzed using GraphPad Prism software (version 7.04) by one-way ANOVA post hoc Tukey-Kramer Multiple Comparisons Test. $p<0.05$ was considered statistically significant.

\section{RESULTS}

Presence of triterpenoids in all extracts was confirmed by violet-blue coloration bands after derivatizing with ASR. However, more intense bands were seen in EtAS as compared to other extracts which dictates, presence of higher amount of triterpenoids in it [Figure 1].

IR spectra of isolated lupeol showed characteristic stretch at 3291, 1636 and 1188 corresponding to $\mathrm{O}-\mathrm{H}$ of alcohols, $\mathrm{C}=\mathrm{C}$ of alkenes and $\mathrm{C}-\mathrm{O}$ of alcohol, ethers or esters respectively [Figure 2]. Similarly, IR spectra of ursolic acid showed characteristic stretch at 3414, 1687 and 1188 corresponding to $\mathrm{O}-\mathrm{H}$ of alcohols, $\mathrm{C}=\mathrm{O}$ of acids and $\mathrm{C}-\mathrm{O}$ of alcohols, ethers or esters respectively [Figure 3]. Also in mass spectrometry, observed molecular weight of isolated pentacyclic triterpenoids matched with the actual molecular weight of lupeol and ursolic acid which dictates their precise isolation by Prep-TLC [Figure 4, 5].

Nitrogenous wastes like creatinine, uric acid, BUN; inflammatory mediators like TNF- $\alpha$ and IL-6 were elevated in serum of CI group; whereas their rise was prevented on administration of EtAS and its isolated pentacyclic triterpenoids. Levels of promoters of crystallization like sodium, potassium, chloride, calcium, oxalate and phosphorus were increased on ingestion 


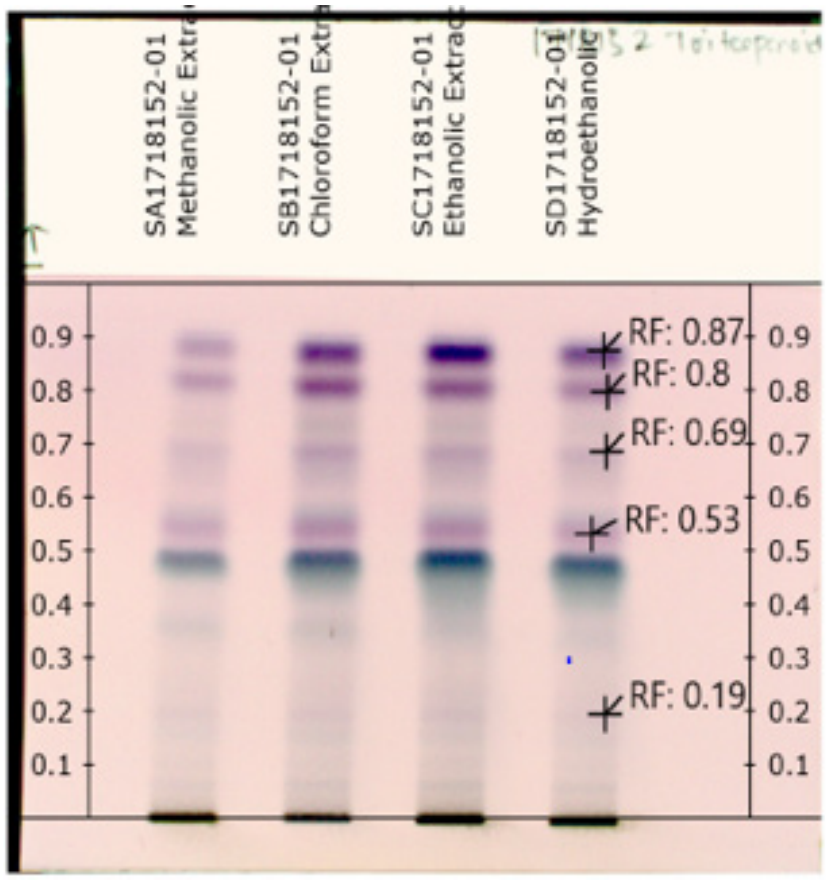

Figure 1: HPTLC chromatogram showing presence of triterpenoids.

HPTLC chromatogram of extracts at $366 \mathrm{~nm}$ showing different bands of separated triterpenoids.

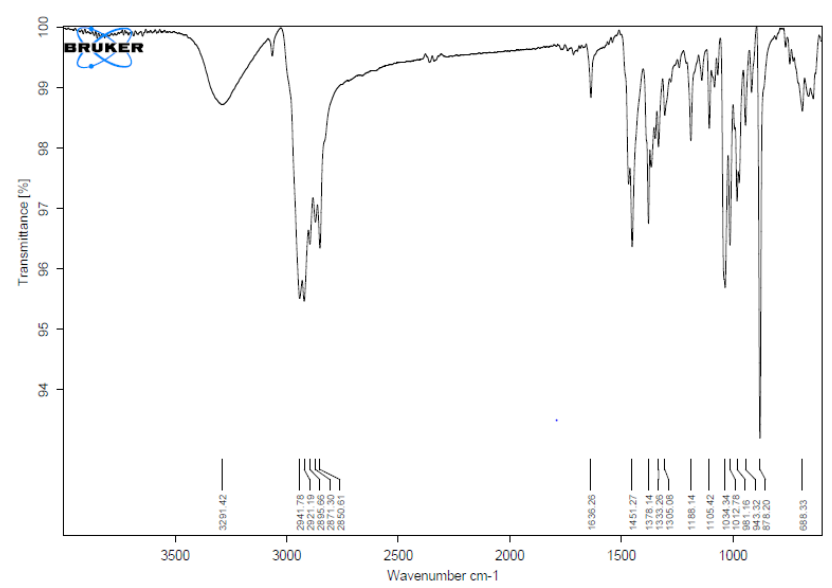

Figure 2: IR spectra of isolated lupeol.

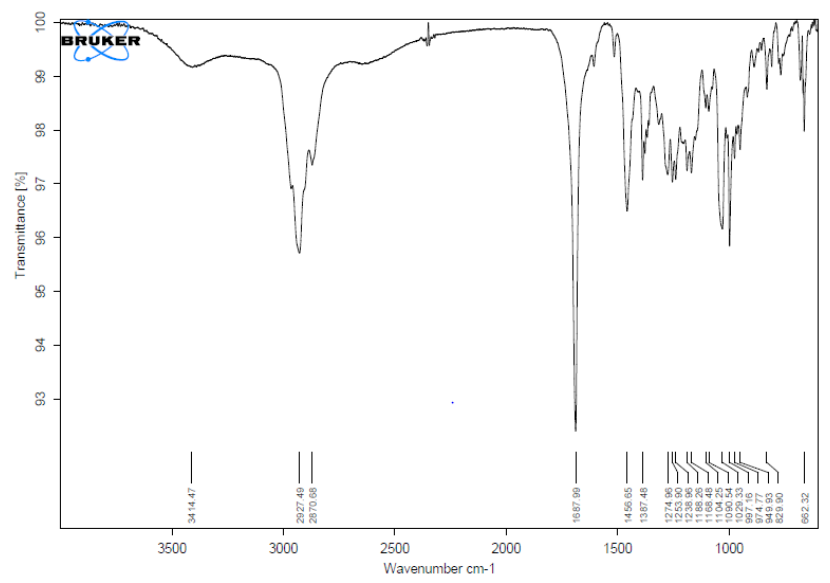

Figure 3: IR spectra of isolated ursolic acid.

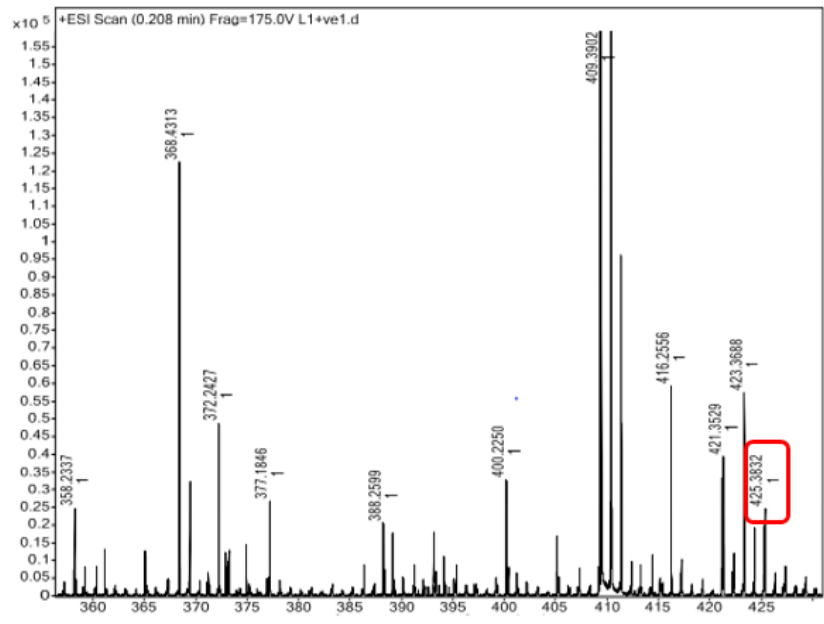

Figure 4: Mass spectra of isolated lupeol.

Observed molecular weight: $425.38 \mathrm{~g} / \mathrm{mol}$. Actual molecular weight: $426.73 \mathrm{~g} / \mathrm{mol}$.

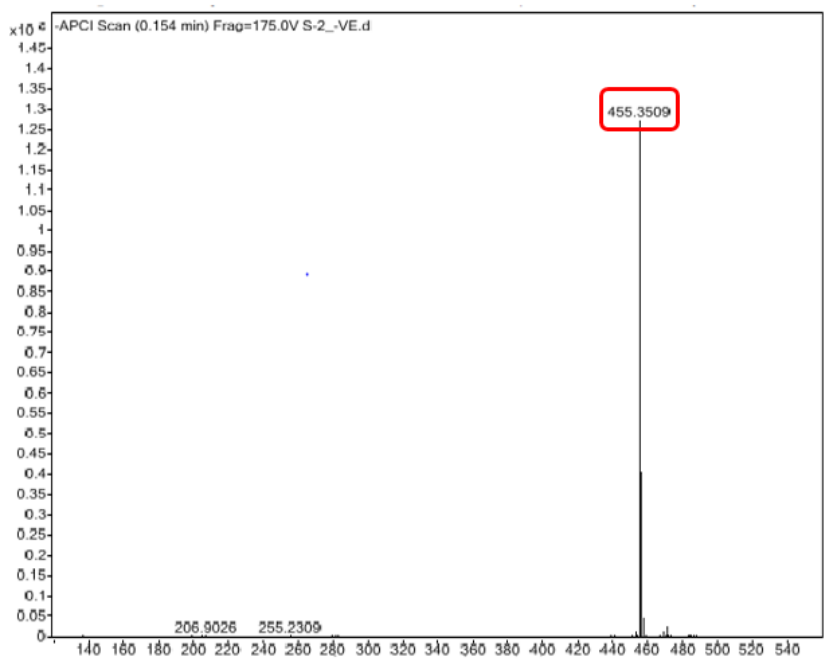

Figure 5: Mass spectra of isolated ursolic acid.

Observed molecular weight: $455.35 \mathrm{~g} / \mathrm{mol}$. Actual molecular weight: $456.71 \mathrm{~g} / \mathrm{mol}$.

of EG whereas rise in their levels was prevented in treatment groups [Table 1].

Urine volume was decreased and weight of kidneys were increased due to accumulation of crystals in CI group, which in turn was prevented in treatment groups. [Table 2]

Microscopic examination of urine revealed presence of numerous stag horn calculi in CI group, whereas urine derived from treatment groups lacked such calculus. [Figure 6]

Histopathological examination of kidneys derived from CI group revealed destructed morphology of nephrons due to deposition of large polymorphic crystals and cystic dilatation of tubules [Figure 7]. Also, IL-6, TNF$\alpha$, calcium, oxalate and malondialdehyde (MDA) levels were increased and SOD, GSH and CAT were decreased in kidney tissue indicating inflammation and oxidative 
Table 1: Effect on biochemical parameters of serum and urine.

\begin{tabular}{|c|c|c|c|c|c|c|c|}
\hline \multicolumn{2}{|c|}{ Biochemical parameters } & \multicolumn{6}{|c|}{ Groups } \\
\hline & & VC & $\mathrm{Cl}$ & TT/CYS & TT/LD & TT/HD & TT/PT \\
\hline \multirow{3}{*}{$\begin{array}{l}\text { Serum } \\
(\mathrm{mg} / \mathrm{dl})\end{array}$} & Creatinine & $0.5 \pm 0.02$ & $1.88 \pm 0.05^{\#}$ & $0.61 \pm 0.01^{*}$ & $0.89 \pm 0.04^{*}$ & $0.61 \pm 0.02^{*}$ & $0.71 \pm 0.03^{*}$ \\
\hline & Uric acid & $1.5 \pm 0.01$ & $3.21 \pm 0.04^{\#}$ & $1.67 \pm 0.02^{*}$ & $1.86 \pm 0.03^{*}$ & $1.67 \pm 0.02^{*}$ & $1.77 \pm 0.02^{*}$ \\
\hline & BUN & $26.88 \pm 0.95$ & $50.47 \pm 1.09^{\#}$ & $31.82 \pm 1.66^{*}$ & $39.55 \pm 0.8^{*}$ & $31.99 \pm 1.2^{*}$ & $36.17 \pm 0.74$ \\
\hline \multirow{3}{*}{$\begin{array}{c}\text { Urine } \\
(\mathrm{mmol} / \mathrm{L})\end{array}$} & Sodium & $49.41 \pm 3.05$ & $92.46 \pm 2.31^{\#}$ & $55.57 \pm 0.69^{*}$ & $63.68 \pm 1.82^{*}$ & $55.76 \pm 1.08^{*}$ & $58.87 \pm 0.97^{*}$ \\
\hline & Potassium & $43.43 \pm 1.24$ & $99.65 \pm 0.61^{\#}$ & $59.45 \pm 0.88^{*}$ & $74.39 \pm 1.41^{*}$ & $58.44 \pm 1.56^{*}$ & $63.26 \pm 2.45^{*}$ \\
\hline & Chloride & $22.84 \pm 0.49$ & $50.53 \pm 0.33^{\#}$ & $27.98 \pm 0.36^{*}$ & $33.37 \pm 0.47^{*}$ & $28.44 \pm 0.97^{*}$ & $30.1 \pm 1.16^{*}$ \\
\hline \multirow{3}{*}{$\begin{array}{l}\text { Urine } \\
\text { (mg/dl) }\end{array}$} & Calcium & $1.42 \pm 0.04$ & $4.78 \pm 0.18^{\#}$ & $1.57 \pm 0.02^{*}$ & $2.36 \pm 0.03^{*}$ & $1.58 \pm 0.02^{*}$ & $1.73 \pm 0.03^{*}$ \\
\hline & Oxalate & $0.47 \pm 0.05$ & $3.21 \pm .06^{\#}$ & $0.58 \pm 0.05^{*}$ & $0.9 \pm 0.08^{*}$ & $0.57 \pm 0.06^{*}$ & $0.67 \pm 0.05^{*}$ \\
\hline & Phosphorus & $7.11 \pm 0.18$ & $12.07 \pm 0.45^{\#}$ & $7.8 \pm 0.36^{*}$ & $9.06 \pm 0.36^{*}$ & $7.96 \pm 0.26^{*}$ & $8.63 \pm 0.21^{*}$ \\
\hline
\end{tabular}

Values are expressed as Mean \pm SD, where $n=6$; Data was analyzed by One-way ANOVA followed by Tukey-Kramer post hoc multiple comparison test, \#p<0.05 was considered statistically significant for $\mathrm{Cl}$ group as compared with VC group; ${ }^{*} p<0.05$ was considered statistically significant for TT/CYS, TT/LD, TT/HD and TT/PT groups as compared with $\mathrm{Cl}$ group

\begin{tabular}{|c|c|c|c|c|}
\hline \multicolumn{5}{|c|}{ Table 2: Effect on tissue inflammatory mediator levels, urine volume and kidney weight. } \\
\hline Groups & TNF- $\alpha$ & IL-6 & Urine Volume (ml/24hr) & Kidney weight (g) \\
\hline VC & $412.06 \pm 4.74$ & $716.2 \pm 6.9$ & $7.85 \pm 0.18$ & $1.51 \pm 0.05$ \\
\hline CI & $695.06 \pm 6.02^{\#}$ & $1106.44 \pm 21.53^{\#}$ & $1.93 \pm 0.19^{\#}$ & $1.99 \pm 0.07^{\#}$ \\
\hline TT/CYS & $422.72 \pm 3.77^{*}$ & $753.77 \pm 5.4^{*}$ & $7.78 \pm 0.23^{*}$ & $1.49 \pm 0.02^{*}$ \\
\hline TT/LD & $549.98 \pm 5.44^{*}$ & $883.92 \pm 7.52^{*}$ & $6.81 \pm 0.23^{*}$ & $1.75 \pm 0.03^{*}$ \\
\hline TT/HD & $422.64 \pm 4.27^{*}$ & $755.53 \pm 7.69^{*}$ & $7.75 \pm 0.28^{*}$ & $1.49 \pm 0.03^{*}$ \\
\hline TT/PT & $431.73 \pm 3.07^{*}$ & $784.93 \pm 6.37^{*}$ & $7.83 \pm 0.16^{*}$ & $1.52 \pm 0.05^{*}$ \\
\hline
\end{tabular}

Values are expressed as Mean $\pm S D$, where $n=6$; Data was analyzed by One-way ANOVA followed by Tukey-Kramer post hoc multiple comparison test, \#p<0.05 was considered statistically significant for $\mathrm{Cl}$ group as compared with VC group; ${ }^{*} p<0.05$ was considered statistically significant for TT/CYS, TT/LD, TT/HD and TT/PT groups as compared with $\mathrm{Cl}$ group.

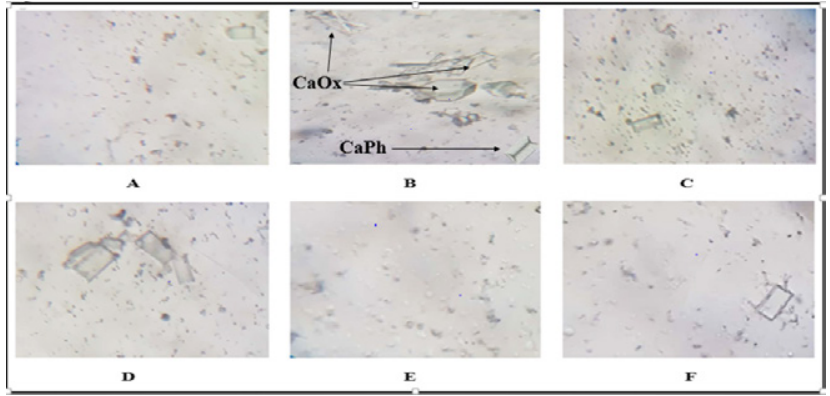

Figure 6: Microscopic examination of urine for presence of crystals at $45 \mathrm{X}$ magnification.

A, B, C, D, E and F represents VC, CI, TT/CYS, TT/LD, TT/HD and TT/PT groups respectively.

$\mathrm{CaOx}$ : Calcium Oxalate; $\mathrm{CaPh}$ : Calcium Phoshate

stress. Kidneys from treatment group exhibited normal morphology with no deposition of crystals. Levels of inflammatory mediator and anti-oxidant enzyme levels were also restored back to normal [Figure 8-10].

\section{DISCUSSION}

Urolithiasis is the pathological condition of genitourinary system; characterized by formation of stony concretions in urinary system resulting in obstruction to the flow
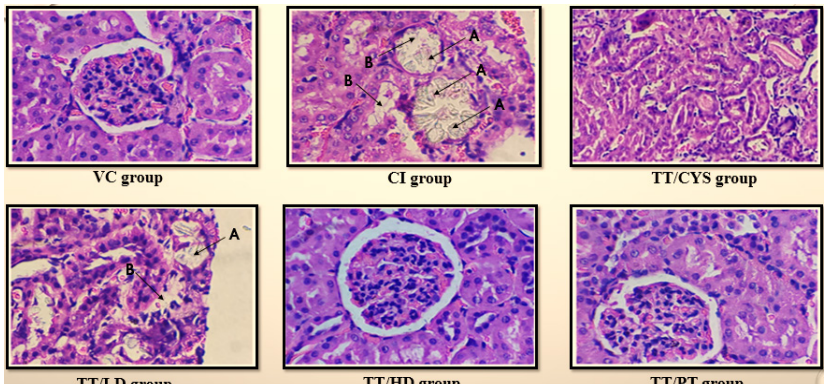

Figure 7: Histopathological examination of kidney sections (X400).

A: large polymorphic and irregular crystal deposits; B: tubular dilatation and damage

of urine, renal colic and urinary tract infections. The prevalence of this global condition has increased over the last few years due to modifications in lifestyle and dietary habits like high intake of food rich in oxalate, sodium, sugars and low intake of fluids due to which urine gets supersaturated with respect to crystal promoting constituents resulting in calculogenesis. ${ }^{14}$

Search of herbal remedy for anti-urolithiatic effect is of primeimportancebecausecurrentmedicalmanagement of urolithiasis is costly, time bound and associated 


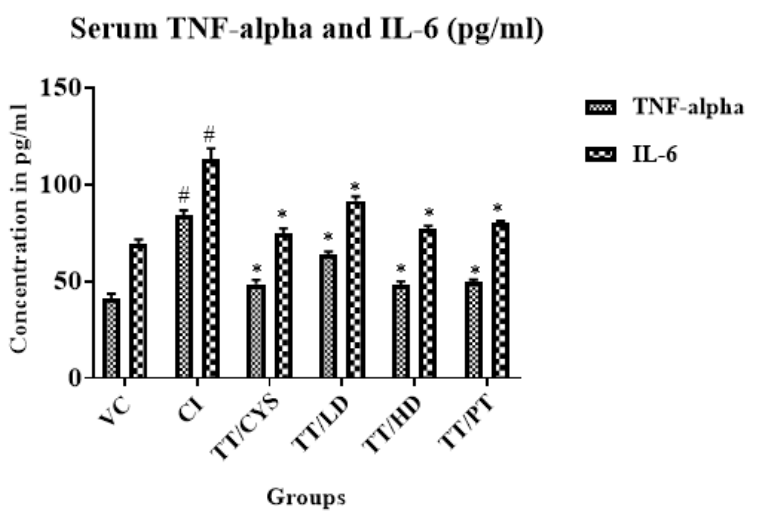

Figure 8: Effect on serum inflammatory mediator levels. Values are expressed as Mean $\pm \mathrm{SD}$, where $n=6$; Data was analyzed by One-way ANOVA followed by Tukey-Kramer post hoc multiple comparison test, $\# p<0.05$ was considered statistically significant for CI group as compared with VC group; ${ }^{*} p<0.05$ was considered statistically significant for TT/CYS, TT/LD, TT/HD and TT/PT groups as compared with CI group

\section{Kidney tissue calcium and oxalate (mg/g)}

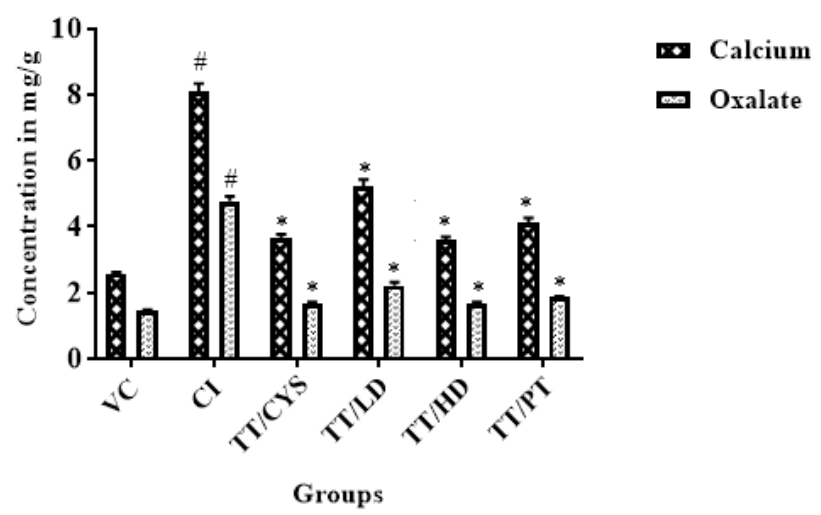

Figure 9: Effect on Kidney tissue calcium and oxalate levels. Values are expressed as Mean $\pm \mathrm{SD}$, where $n=6$; Data was analyzed by One-way ANOVA followed by Tukey-Kramer post hoc multiple comparison test, $\# p<0.05$ was considered statistically significant for CI group as compared with VC group; $" p<0.05$ was considered statistically significant for TT/CYS, TT/LD, TT/HD and TT/PT groups as compared with CI group

with substantial side effects. Herbs are loaded with physiologically active phytoconstituents possessing vital potential in management of various ailments. Pentacyclic triterpenoids of plant origin have been claimed to be effective in preventing crystal induced renal per oxidative changes and diluting urinary stone forming constituent ${ }^{5}$ which forms the basis for selection of $A$. scholaris in this study. Presence of triterpenoids in all extracts and greater amount in EtAS was confirmed by HPTLC study. Thus, EtAS may carry vital therapeutic potential for treatment of urolithiasis and hence subjected to in vivo studies.

EG was used to induce urolithiasis. Kidney is the major target organ for toxicity by EG. ${ }^{15}$ Chronic oral administration of EG $(0.75 \% \mathrm{v} / \mathrm{v})$ for 14 days results
Kidney tissue anti-oxidant levels

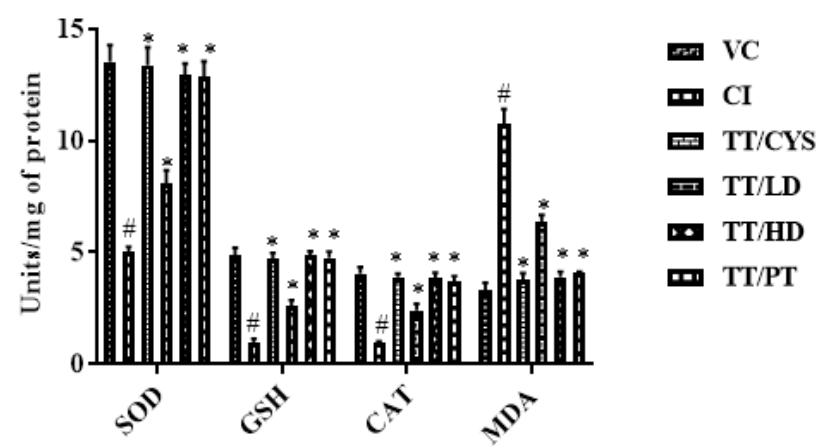

Anti-oxidant enzymes

Figure 10: Effect on Kidney tissue anti-oxidant levels. SOD: Superoxide dismutase; GSH: Glutathione; CAT: Catalase; MDA: Malondialdehyde

Values are expressed as Mean $\pm \mathrm{SD}$, where $n=6$; Data was analyzed by One-way ANOVA followed by Tukey-Kramer post hoc multiple comparison test, $\# p<0.05$ was considered statistically significant for $\mathrm{CI}$ group as compared with VC group; ${ }^{*}<<0.05$ was considered statistically significant for TT/CYS, TT/LD, TT/HD and TT/PT groups as compared with CI group.

in formation of renal calculi composed mainly of $\mathrm{CaOx} \cdot{ }^{16} \mathrm{EG}$ is rapidly absorbed and metabolized in the liver via alcohol or aldehyde dehydrogenase to glycolic acid. Glycolic acid is oxidized to glyoxylic acid, which in turn is further oxidized to oxalic acid by glycolate oxidase. High doses of EG when given as an oral bolus causes saturation dependent accumulation of glycolic acid in plasma; ${ }^{17}$ this in turn increases oxalic acid content in urine which ionizes to oxalate and then undergoes precipitation reaction with calcium ions to form $\mathrm{CaOx}$. Oxalate induces lipid peroxidation resulting in renal epithelial injury which exposes variety of crystal adhesion molecules on epithelial surface promoting crystal retention and stone formation. ${ }^{17}$ These crystals further interacts with the renal tissue causing inflammation, generating reactive oxygen species and depleting antioxidant enzymes. Thus in CI group levels of inflammatory mediators were found to be elevated in serum and kidney homogenate; whereas antioxidant enzyme levels were significantly $(p<0.001)$ declined indicating lipid peroxidation in CI group. Treatment with EtAS and its isolated pentacyclic triterpenoids prevented rise in levels of inflammatory mediators and restored antioxidant enzymes thus exhibiting antioxidant ${ }^{18}$ and anti-inflammatory ${ }^{19}$ effects of leaves of $A$. scholaris. Treatment would have also prevented metabolism of EG to oxalate and rise in the ionic pattern of urine there by preventing formation of $\mathrm{CaOx}$ crystals and resulting inflammatory tissue damage.

Due to obstruction to the outflow of urine by deposited crystals GFR decreased and nitrogenous waste was accumulated in blood ${ }^{20}$ and the excreted urine volume 
was decreased in CI group. EtAS and its isolated pentacyclic triterpenoids significantly $(p<0.001)$ prevented rise in these nitrogenous waste levels and any alterations in micturition volume. Change in the ionic pattern of urine is one of the major determinant of stone formation. Rats in CI group excreted significantly $(p<0.001)$ higher amount of electrolytes which is indicator of urolithiasis. Microscopic examination of urine revealed presence of large and abundant crystals in CI group. Whereas EtAS and its isolated pentacyclic triterpenoids significantly $(p<0.001)$ prevented perturbations in ionic pattern of urine, thus preventing crystal formation.

Microscopic examination of kidney sections derived from CI group disclosed deposition of irregular polymorphic crystals in glomerulus and tubules. Morphology of glomerulus and tubules was severely destructed due to interstitial inflammation and dilatation of tubules, which was evident from appearance of void spaces in the tissue section. Kidneys derived from TT/LD group revealed less and small size calculi with minimum tubular damage and dilatation as compared to CI group. Kidneys from TT/HD and TT/PT groups had normal morphology with no calculi, thus indicating effective prevention in formation of any calculus.

Urolithiasis is associated with renal colic; excruciating pain due to movement of calculi across the tract, for which use of analgesics are mandatory. These OTC analgesics bring array of side effects making their regular consumption risky. Other etiological factor promoting recurrence is frequent urinary tract infections. As leaves of $A$. scholaris are reported to possess analgesic ${ }^{19}$ and anti-microbial properties ${ }^{21}$ treatment with it can overcome the need for multiple drug therapy to treat associated complications. Thus multifaceted effects of $A$. scholaris and its pentcyclic triterpenoids proves to be effective treatment for prevention of urolithiasis.

\section{CONCLUSION}

Leaves of $A$. scholaris contains significant amount of pentacyclic triterpenoids possessing anti-inflammatory and antioxidant potentials to prevent urolithiasis and its associated complications. Thus in present study array of pharmacological effects exhibited by $A$. scholaris and its pentacyclic triterpenoids viz. lupeol and ursolic acid establishes a potent herbal remedy for prevention and management of urolithiasis.

\section{ACKNOWLEDGEMENT}

We acknowledge the facility under DST-FIST Funding (Letter SR/FST/College-264 dated 18th November,
2015) for IR spectra, Biochemical analyzer-ERBA chem-7 (Transasia, Germany), Microplate reader-ELx800 and Microplate washer-ELx50 (Biotek Instruments).

We also acknowledge the necessary facility provided by Anchrom Laboratory (Mulund, India) for carrying out HPTLC analysis.

\section{CONFLICT OF INTEREST}

The authors declare that there is no conflict of interest.

\section{ABBREVIATIONS}

ASR: Anisaldehyde Sulphuric acid Reagent; $A$. Scholaris: Alstonia scholaris; ANOVA: Analysis of variance; CaOx: Calcium oxalate; ChAS: Chloroform extract of Alstonia scholaris; CPCSEA: Committee for purpose of control and supervision on animals; CMC: Carboxymethly Cellulose; CI: Calculi Induced; CAT: Catalase; ESWL: Extracorporeal Shockwave Lithotripsy; EtAS: Ethanolic extract of Alstonia scholaris; EG: Ethylene glycol; GFR: Glomerular Filtration Rate; GSH: Glutathione; HeAS: Hydro-ethanolic extract of Alstonia scholaris; $\mathbf{H} \& \mathbf{E}$ stain: Hematoxylin and eosin stain; HCl: Hrdrochloric acid; IR spectroscopy: Infrared spectroscopy; IAEC: Institutional Animal Ethics Committee; IL-6: Interleukin; MET: Medical Expulsive Therapy; MeAS: Methanolic extract of Alstonia scholaris; MDA: Malondialdehyde; NIH: National Institute of Health; NBF: Neutral Buffered Formalin; OTC: Over the counter; SOD: Superoxide dismutase; TT/CYS: Treatment with Cystone; TT/LD: Treatment with low dose of extract; TT/HD: Treatment with high dose of extract; TT/ PT: Treatment with isolated pentacyclic triterpenoid; TNF- $\alpha$ : Tumor necrosis factor- $\alpha$; VC: Vehicle Control.

\section{REFERENCES}

1. Sunitha J, Asha S, Taju G. Protective effect of spirulina on ethylene glycol induced urolithiasis in rats. Int Res J Pharm. 2012;3(5):444-8.

2. Prien EL, JrPrien EL. Composition and structure of urinary stone. Am J Med. 1968;45(5):654-762.

3. Kishimoto T, Yamamoto K, Sugimoto T, Yoshihara H, Maekawa M. Side effects of extracorporeal shock-wave exposure in patients treated by extracorporeal shock-wave lithotripsy for upper urinary tract stone. Eur Urol. 1986;12:30813.

4. Uribarri J, Oh MS, Carroll HJ. The first kidney stone. Ann Intern Med. 1989;111(12):1006-9.

5. Malini MM, Lenin M, Varalakshmi P. Protective effect of triterpenes on calcium oxalate crystal-induced peroxidative changes in experimental urolithiasis. Pharmacol Res. 2000;41(4):413-8.

6. Wang CM, Chen HT, Wu ZY, Jhan YL, Shyu CL, Chou CH, et al. Antibacterial and synergistic activity of pentacyclic triterpenoids isolated from Alstonia scholaris. Molecules. 2016;21(2):139.

7. Bano H, Jahan H, Makbul SAA, Kumar BN, Husain S, Sayed A, et al. Effect of Piper cubeba L. fruit on ethylene glycol and ammonium chloride induced urolithiasis in male Sprague dawley rats. Integr Med Res. 2018;7(4):358-65. 
8. Hodgkinson A, Williams A. An improved colorimetric procedure for urine oxalate. Clin Chim Acta. 1972;36(1):127-32.

9. Dixit $\mathrm{P}$, Koti BC, Vishwanathswamy AHM. Antiurolithiatic activity of crashcal on ethylene glycol induced urolithiasis in rats. RGUHS J Pharm Sci. 2014;4(1):30-5.

10. Sun M, Zigman S. An improved spectrophotometric assay for superoxide dismutase based on epinephrine auto-oxidation. Anal Biochem. 1978;90(1):81-9.

11. Sinha AK. Calorimetric Assay of Catalase. Anal Biochem. 1972;47(2):389-94.

12. Ellman GL. Tissue sulphydryl groups. Arch Biochem Biophys. 1959;82(1):70-7.

13. Okhawa H, Ohishi N, Yagi K. Assay for Lipid Peroxides in animal tissues by thiobarbituric acid reaction. Anal Biochem. 1979;95(2):351-8.

14. Bahuguna Y, Rawat MSM, Juyal V, Gupta V. Antilithiatic effect of flowers of Jasminum auriculum Vahl. Int J Green Pharm. 2009;155-9.

15. Schladt L, Ivens I, Karbe E, Ruhl-Fehlert C, Bomhard E. Subacute oral toxicity of tetra ethylene glycol and ethylene glycol administered to wistar rats. Exp Toxicol Pathol. 1998;50(3):257-65.

\section{PICTORIAL ABSTRACT}
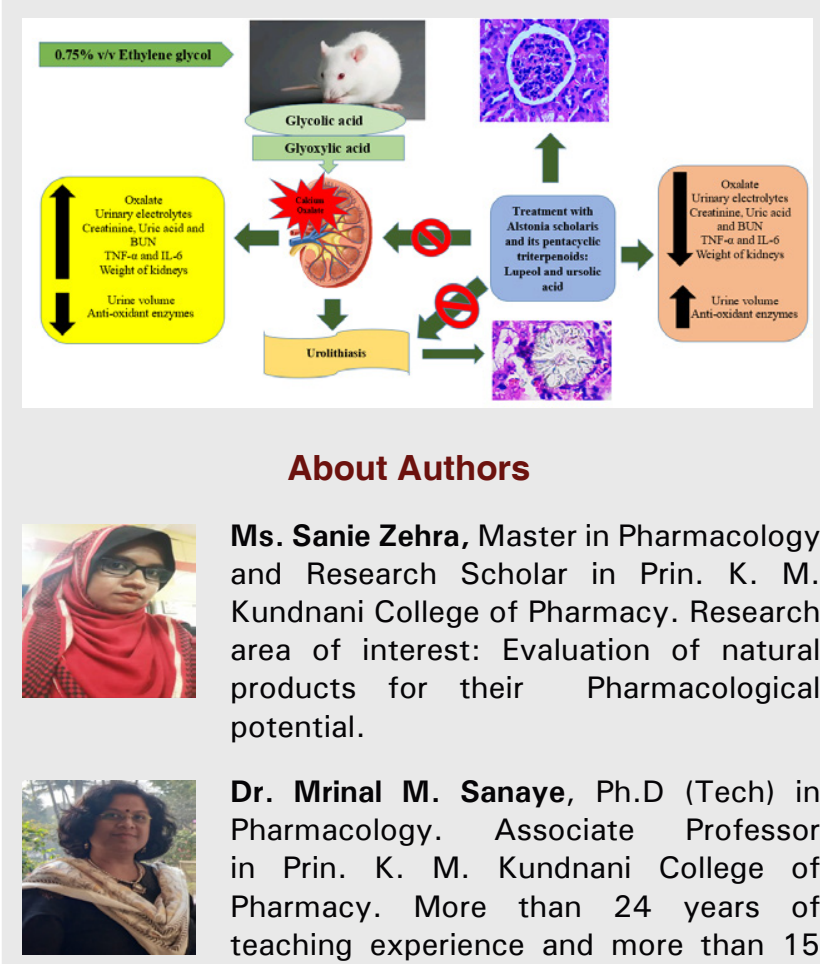

\section{About Authors}

Ms. Sanie Zehra, Master in Pharmacology and Research Scholar in Prin. K. M. Kundnani College of Pharmacy. Research area of interest: Evaluation of natural products for their Pharmacological potential.

Dr. Mrinal M. Sanaye, Ph.D (Tech) in Pharmacology. Associate Professor in Prin. K. M. Kundnani College of Pharmacy. More than 24 years of teaching experience and more than 15 years of research experience. Areas of interest: Neuropharmacological, cardiovascular, metabolic syndrome, urolithiasis and osteoporosis studies.
16. Huang HS, Ma MC, Chen J, Chen CF. Changes in the oxidant-antioxidant balance in the kidney of rats with nephrolithiasis induced by ethylene glycol. J Urol. 2002;167(6):2584-93.

17. Thangarathinam N, Jayshree N, Vijay MA, Ramanathan L. Effect of polyherbal formulation on ethylene glycol induced urolithiasis. Int J Pharm Pharm Sci. 2013;5(3):994-7.

18. Antony M, Menon DB, Joel J, MS DL, Arun K, Thankamani V. Phytochemical analysis and antioxidant activity of Alstonia scholaris. Phcog J. 2011;3(26):13-8.

19. Shang JH, Cai $X H$, Feng $T$, Zhao $Y L$, Wang JK, Zhang LY, et al. Pharmacological evaluation of Alstonia scholaris: Anti-inflammatory and analgesic effects. J Ethnopharmacol. 2010;129(2):174-81.

20. Atmani F, Slimani Y, Minouni M, Hacht B. Prophylaxis of calcium oxalate stones by Hemiaria hirsute on experimentally induced nephrolithiasis in rats. BJU Int. 2003;92(1):137-40.

21. Parcha V, Ghosh B, Nair A, Mittal R. Antimicrobial activity of Alstonia scholaris leaf extracts. Indian Drugs. 2003;40(7):412-3.

\section{SUMMARY}

Conventional allopathic drugs used for treatment of urolithiasis involves multiple drug therapy with several side effects and increased cost. Herbs with array of therapeutic effects thus can be used as safe alternative or adjunct therapy for urolithiasis. Naturally occurring pentacyclic triterpenes of plant origin are reported to possess wide range of pharmacological effects. Lupeol has been found to be effective in reducing the risk of stone formation in animals and Alstonia scholaris being rich source of lupeol is selected in present study to assess its antiurolithiatic effect against ethylene glycol-induced urolithiasis rat model. This study involves quantification, isolation and characterization of pentacyclic triterpenoids (lupeol and ursolic acid) from leaves of $A$. scholaris. Among series of extracts extracted with solvents of variable polarity, ethanolic extract (EtAS) was found to contain highest amount of pentacyclic triterpenoids and hence selected for in vivo study. On preclinical evaluation of EtAS and lupeol it was observed that perturbations in levels of nitrogenous wastes (Creatinine, BUN and uric acid), inflammatory mediators (TNF- $\alpha$ and IL-6) and urinary electrolytes (Sodium, potassium, chloride, oxalate, calcium and phosphorus) were significantly prevented. Treatments also significantly reduced oxidative stress and thus associated inflammation thereby preventing formation of renal calculi. Histopathological studies revealed decreased renal damage and deposition of crystals along with attenuated excretion of crystals in urine. Thus present study indicates promising antiurolithiatic activity of Alstonia scholaris which might be due to presence of rich amount of pentacyclic triterpenoids. This study thus provides an effective herbal therapy for treatment and management of urolithiasis.

Cite this article: Zehra S, Sanaye MM. Evaluation of Anti-urolithiatic Potential of Leaves of Alstonia scholaris and its Isolated Pentacyclic Triterpenoids in Ethylene Glycol-induced Renal Calculi Rat Model. Indian J of Pharmaceutical Education and Research. 2021;55(1):232-9. 